\title{
Inhibition of Streptococcus pneumoniae autolysins highlight distinct differences between chemical and genetic inactivation
}

\begin{abstract}
Brad A Haubricha,d\#, Saman Nayyaba,e\#, Caroline Williams ${ }^{a}$, Andrew Whitman ${ }^{\mathrm{a}}$, Tahl Zimmermanc,
\end{abstract} Qiong Lif, Yuxing Chen ${ }^{f}$, Cong-Zhao Zhou ${ }^{f}$, Amit Basu ${ }^{b^{*}}$, Christopher W Reida ${ }^{*}$

[a] Center for Health and Behavioural Sciences

Dept. of Science and Technology

Bryant University

1150 Douglas Pike, Smithfield, RI 02917

*Corresponding authors E-mail: creid@bryant.edu, abasu@brown.edu

\# both authors contributed equally to this work

[b] Dept of Chemistry

Brown University

Providence, RI

[c] Dept. of Family and Consumer Sciences

North Carolina A\&T State University

Greensboro, NC

[d] Touro University Nevada,

College of Osteopathic Medicine, Dept of Basic Sciences

Henderson, NV, 89014

[e] University of Massachusetts Amherst

Dept of Molecular and Cellular Biology

230 Stockbridge Rd

Amherst, MA

[f] School of Life Sciences

University of Science and Technology of China

Hefei, Anhui, 230027, P.R. China

\begin{abstract}
:
Despite renewed interest, development of chemical biology methods to study peptidoglycan metabolism has lagged in comparison to the glycobiology field in general. To address this, a panel of diamides were screened against the Gram-positive pathogen Streptococcus pneumoniae to identify inhibitors of bacterial growth. The screen identified the diamide fgkc as a narrow spectrum bacteriostatic inhibitor of $S$. pneumoniae growth with an MIC of $7.8 \mu \mathrm{M}$. The diamide inhibited detergent-induced autolysis in a concentration dependent manner indicating peptidoglycan degradation as the mode-of-action. Genetic screening of autolysin mutants suggested LytB, an endo-N-acetylglucosaminidase, involved in cell division as the potential target. Surprisingly, biochemical, and phenotypic analysis contradicted the genetic screen
\end{abstract}


results. Phenotypic studies with the $\Delta l y t B$ strain illustrate the difference between genetic and chemical inactivation of autolysins. These findings suggest that meta-phenotypes including autolytic activity, cell morphology, and genetic screening can be the result of the complex interaction of one or more possible pathways that are connected to cell wall metabolism.

\section{Introduction}

Antibiotic resistance is a growing global threat. Drug-resistant Streptococcus pneumoniae alone is estimated to cause 1.2 million infections with an excess of 96 million USD in medical costs per annum.(1) In light of this, there is need for the development of new therapeutics.

Peptidoglycan (PG) is the primary structural heteropolymer conferring strength and cell shape determination in both Gram-negative and Gram-positive organisms (Figure 1). The PG backbone is composed of $\beta-1,4$-linked $\mathrm{N}$-acetylmuramic acid (MurNAc) and $\mathrm{N}$ acetylglucosamine (GlcNAc). Attached to the C-3 lactyl moiety of MurNAc is a stem peptide that is involved in cross-linking the adjacent glycan strands to form the three-dimensional structure. The incorporation of new material into the existing cell wall requires the delicate homeostasis of biosynthetic and degradative enzymes to bring new material into the stress bearing layer to prevent lysis. $(2,3)$ Disruption of this interplay between degradative and biosynthetic processes via chemical inhibition could provide unique insights into their biological role. Deciphering the exact biological role of autolysins has been a formidable task as functional redundancy complicates attribution of biological roles. Recent biophysical $(3,4)$ and computational studies(5) of bacterial autolysins have begun to unravel their roles in the release of stress in the cell wall to allow for incorporation of new material. A renaissance in peptidoglycan metabolism research has started to provided new chemical biology tools to study synthesis (6) and further insight into membrane mediated steps (7-9) and the role endopeptidases play in methicillin resistance.(10) While the cell wall and peptidoglycan in particular have provided a wealth of clinically relevant antimicrobial targets (11), our understanding of the complex interplay between degradative and synthetic steps is still developing.

Previously, we had carried out a screen of 21 diamides for anti-bacterial activity against the Grampositive Bacillus subtilis. This screen identified the diamide fgkc (Figure 1 inset) as a bacteriostatic inhibitor of Bacillus subtilis growth that targets the major active $N$ acetylglucosaminidase (GlcNAcase) LytG in vitro.(12) Here we report on the screening of this panel of diamides against $S$. pneumoniae, identifying fgkc as a bacteriostatic inhibitor of cell 
growth. A series of subsequent mode-of-action studies in S. pneumoniae highlights distinct differences between chemical and genetic inactivation of autolysins.

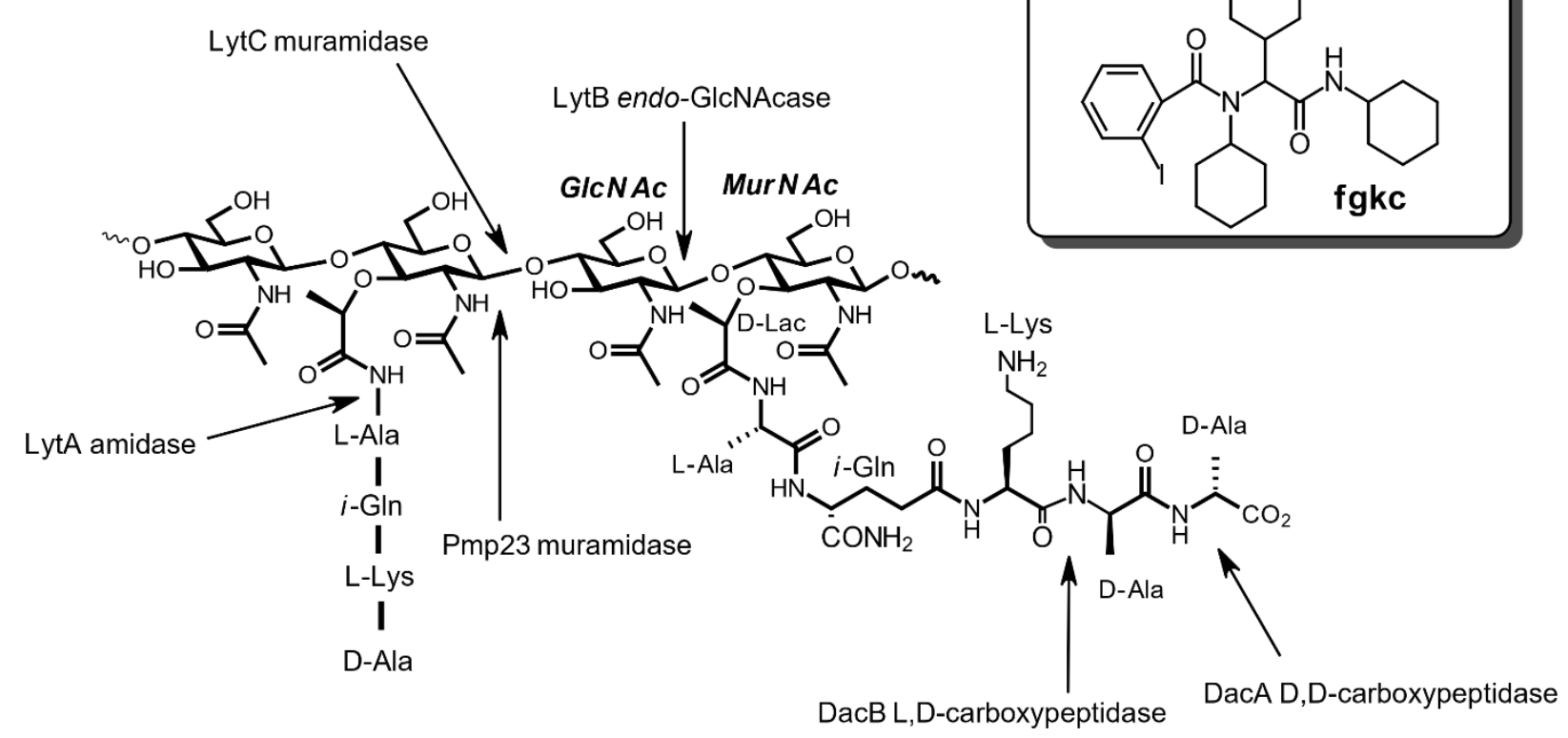

Figure 1. Structure of peptidoglycan showing the cleavage sites of several of the characterized autolysins in S. pneumoniae. Inset: structure of the antimicrobial diamide fgkc.

\section{Materials and Methods}

Strains and compounds. Streptococcus pneumoniae 6305 and R6 were purchased from ATCC (Mannassas, VA), and S. pneumoniae IU1945 ( $\Delta / y t B, \Delta l y t C, \Delta d a c A, \Delta d a c B, \Delta p m p 23, \Delta p b p 1 a$ ) mutants were kindly provided by Dr. Malcolm E. Winkler at the University of Indiana.(13) $S$. pneumoniae TIGR4 and TIGR4 $\Delta$ lytB strains were previously reported.(14) S.pneumoniae strains were grown in Mueller Hinton (MH) broth (Sigma Aldrich, St. Louis, MO) supplemented with $5 \%(\mathrm{v} / \mathrm{v})$ sheep blood (Lampire Biological, Pipersville PA) or MH agar plates containing 1.5 $\%(\mathrm{~m} / \mathrm{v})$ Bacto agar and $5 \%(\mathrm{v} / \mathrm{v})$ sheep blood at $37^{\circ} \mathrm{C}$. Staphylococcus aureus was grown in $\mathrm{MH}$ broth or on solid media, Clostridiodes difficile was grown in brain heart infusion (BHI) and Escherichia coli DH5a on Lauria Burtani (LB) broth or solid media.

Diamide inhibitors were synthesized as described previously.(12) Other reagents, unless otherwise specified were purchased from Sigma (St. Louis, MO). 
MIC assays. MIC values were determined using the resazurin method. $(15,16)$ Briefly, second passage cells of S. pnuemoniae 6305, TIGR4, or R6 were grown in MH broth, and standardized to an $\mathrm{OD}_{600 \mathrm{~nm}}=0.4$. Inhibitors were analyzed via serial dilution into PBS media in microtitre plates. Microtitre plates were inoculated with a $1 / 20$ dilution of the $\mathrm{OD}_{600 \mathrm{~nm}}=0.4$ cell culture with a final concentration of $1 \%$ DMSO. Cultures were grown statically for $24 \mathrm{~h}$ at $37^{\circ} \mathrm{C}$, followed by addition of $30 \mu \mathrm{L}$ of a $0.01 \%(\mathrm{~m} / \mathrm{v})$ solution of resazurin. The plates were incubated for $15 \mathrm{~min}$ to allow stabilization of color production. MICs were read directly off the plate; MICs were recorded as the lowest concentration that inhibited growth. MIC assays with $S$. aureus were performed in $\mathrm{MH}$ broth, $C$. difficile in $\mathrm{BHI}$ broth, while $E$. coli was performed in LB.

Morphological studies of $S$. pnuemoniae. Cultures were prepared from second passage of $S$. pneumoniae 6305, R6 and $\Delta / y t B(13)$ as previously described for MIC determination. Cells were chemically fixed in 20 mM HEPES pH 6.8 containing 1\% formaldehyde. (17) Samples were fixed overnight at $4{ }^{\circ} \mathrm{C}$ to limit de novo cell wall biosynthesis during fixation. Samples were stained with $0.1 \%$ methylene blue (solution in $20 \%$ ethanol). Samples were gently heated to 60 ${ }^{\circ} \mathrm{C}$ for $15-20$ min to bring cells to a common focal plane. Samples were visualized using brightfield microscopy with a Zeiss Primo Star microscope at 1000× magnification. Micrographs were acquired using an Axiocam ERc5s camera and Zen lite software.

Autolysis assays. Cellular autolysis assays were performed as previously described by Cornett and Shockman.(18) Briefly, S. pneumonia 6305 were grown in $\mathrm{MH}$ broth containing 5\% (v/v) defibrinated sheep blood under anaerobic conditions. Cells were harvested by centrifugation (8,000 rpm, $5 \mathrm{~min}$ ) and washed with PBS. Cells were suspended in PBS and autolysis induced with the addition of Triton X-100 to a final concentration of $0.1 \%(\mathrm{v} / \mathrm{v})$ and turbidity monitored at $600 \mathrm{~nm}$ over $60 \mathrm{~min}$. Rates were calculated using the linear portion of the autolysis curves with the rate of autolysis in the absence of inhibitor set at $100 \%$.

Chain dispersing assay. Dispersion of the $\Delta / y t B$ chain morphology with purified LytB was carried out as previously described using the TIGR4 and associated $\Delta / y t B$ strains.(14) LytB was added to the cell suspension at a final concentration of $2 \mu \mathrm{M}$. The final concentration of fgkc in the assays was $40 \mu \mathrm{M}$.

DNA intercalation assays. To determine if fgkc is a DNA intercalator, DNA mobility shift assays were performed as previously described using BamHI-linearized pUC18 plasmid.(19) The known DNA intercalator actinomycin D was used as a control. 
DNase I assays. Degradation of the pUC18 plasmid DNA was assayed using $150 \mathrm{ng}$ of linearized pUC18 plasmid.(20) Compound titrations in DMSO were added and reactions were initiated with 0.002 units of DNase I. The reactions were incubated for $15 \mathrm{~min}$ at $37^{\circ} \mathrm{C}$ before being subjected to agarose gel electrophoresis. EDTA was used as a control for DNase I inhibition.

Lipotechoic acid detection by Western blot. Lipoteichoic acid profiles were analyzed as previously described.(21) Briefly, S. pneumoniae R6 cells were cultured to saturation, harvested $(3000 \times \mathrm{g})$, resuspended in $6 \mathrm{M}$ urea, and incubated at $37^{\circ} \mathrm{C}$ for $5 \mathrm{~min}$ to solubilize proteins. Samples were standardized by total protein content and separated by SDS-PAGE (16\%) and transferred to PVDF membrane. The membrane was incubated with a 1:5000 $\alpha$-phosphocholine monoclonal antibody (SSI Biotech, Santa Cruz, CA). Blots were analyzed by chemiluminescence.

\section{Results and Discussion}

We screened a previously reported (12) panel of 21 diamides against $S$. pneumoniae using the resazurin microtiter assay (Figure S1).(16) Of the 21 compounds screened, fgkc was identified as a single digit micromolar inhibitor of $S$. pneumoniae growth with an MIC of $7.8 \mu \mathrm{M}$ against strains 6305, R6, and TIGR4. Analysis of the mode of growth inhibition demonstrated that fgkc was bacteriostatic. These results for fgkc are comparable to those obtained against $B$. subtilis $(\mathrm{MIC}=3.8 \mu \mathrm{M}) .(12)$ To assess the antimicrobial spectrum of the compound, we screened fgkc against the Gram-positive organisms Clostridiodes difficile, Staphylococcus aureus and the Gram-negative Escherichia coli. In all cases, no antimicrobial activity was observed up to 200 $\mu \mathrm{M}$ suggesting that $\mathbf{f g k c}$ is a narrow spectrum inhibitor of Gram-positive growth. Extrapolating from $B$. subtilis activity where fgkc inhibits LytG, a glycosyl hydrolase family 73 (GH73) enzyme, we presumed the target was a member of $\mathrm{GH} 73$. To date, $S$. pneumoniae possesses one enzyme classified as GH73 (www.cazy.org) - LytB, a cell division associated endo- $\beta$ GlcNAcase belonging to cluster 4 of GH73.(14, 22-24) In contrast, $B$ subtilis LytG is an exoacting GlcNAcase active in vegetative growth belonging to GH73 cluster 2. (25) 
A
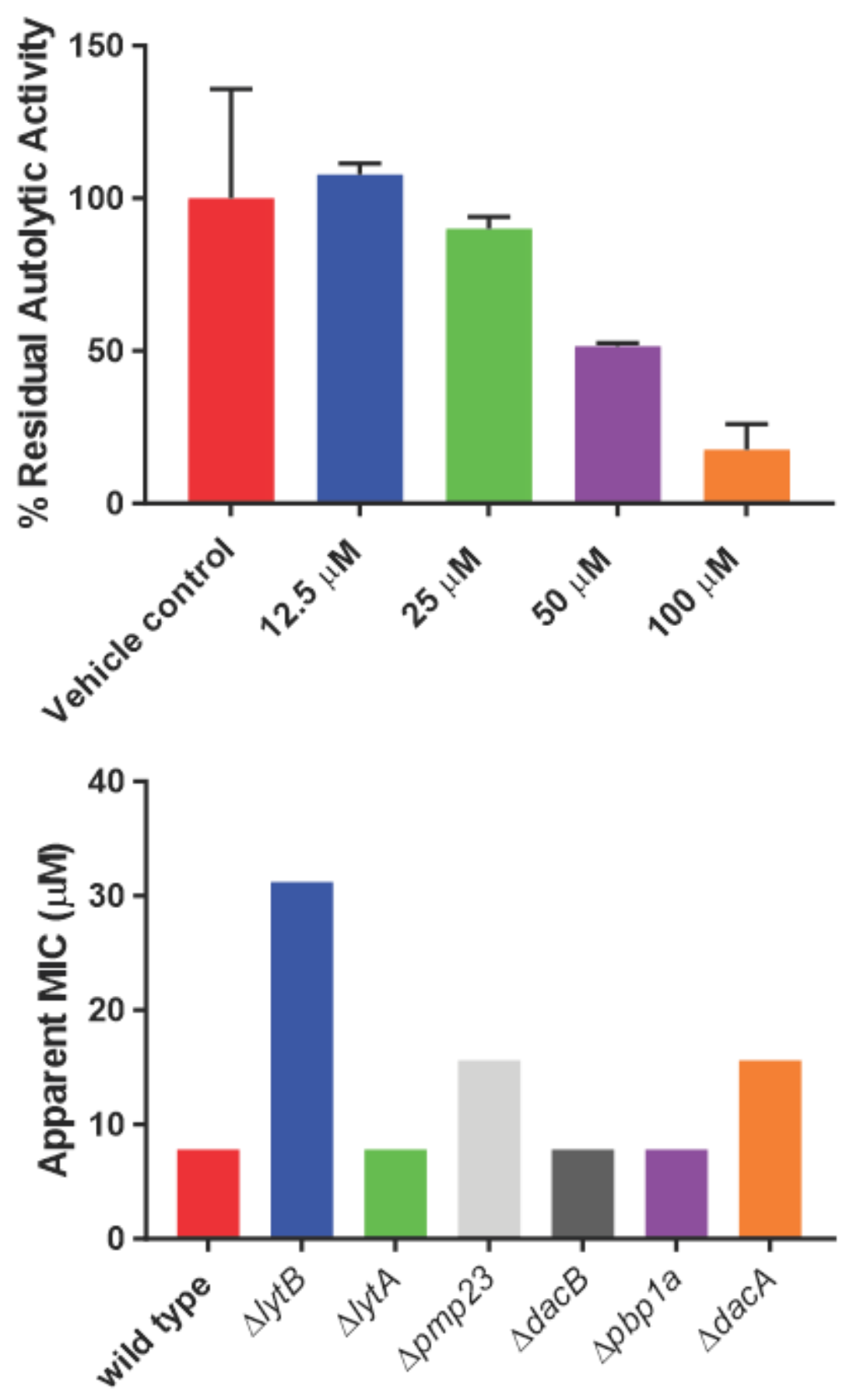

Figure 2 Screening of the diamide fgkc against Streptococcus pneumoniae. (A) The diamide fgkc inhibits detergent-induced autolysis. (B) Genetic screen of S. pneumoniae R6 autolysin and cell wall biosynthesis mutants (13) to identify the target of fgkc. Data shown is the average of experiments performed in biological and technical triplicate. 
Based on our previous findings in B. subtilis, we examined whether fgkc inhibited autolytic activity in S. pneumoniae (Figure 2A). Non-ionic detergent (Triton X-100) induced autolysis of $S$. pneumoniae was inhibited in a concentration induced manner by fgkc, suggesting that this compound does indeed target the cell wall and autolysins specifically. We followed up with a genetic screen of cell wall autolysins (Figure 1) and PG biosynthetic enzyme knockout strains to further refine the mode-of-action and identify the molecular target (Figure 2B). Conventional wisdom in this type of experiment would presume that deletion of the target should result in a reduction in sensitivity to the compound. The genetic screen demonstrated a nearly 4 -fold decrease in sensitivity to fgkc in the $\Delta / y t B$ mutant (13), strongly suggesting LytB was the putative target of the diamide.

To further explore these results, morphological changes induced by sub-MIC concentrations of fgkc and antibiotics of known modes-of-action were investigated (Figure 3). S. pneumoniae treated with fgkc showed a change from the wild-type diploid morphology to a clumping phenotype like the cell wall acting antibiotics bacitracin and vancomycin. The morphology of sub-MIC treated cells showed a deviation from the typical coccoid shape and size. The fgkc induced phenotype did not correlate with the reported phenotypes of a $\Delta / y t B$ mutant, which exhibit a chaining phenotype(14, 26, 27) A clumping phenotype was also observed with subMIC kanamycin. This phenotype has been associated with antibiotics that target intracellular protein synthesis.(28) Additionally, lipoteichoic acid (LTA) disruption was monitored by Western blot in the presence of sub-MIC fgkc. Results indicated that changes to LTA incorporation in the cell wall was not a contributor to the observed fgkc-induced phenotype (Figure S2). In summary, the morphological changes are consistent with the hypothesis that fgkc is acting on a cell wall target. 


\section{Control}

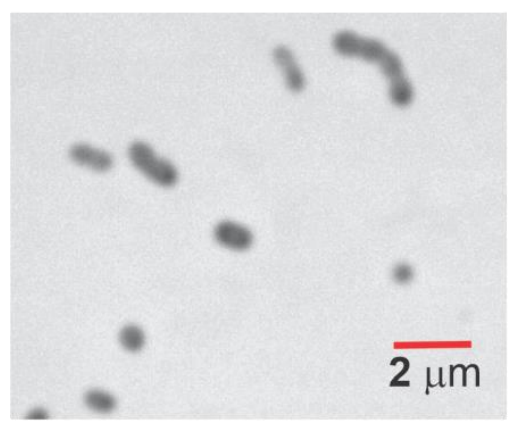

Vancomycin

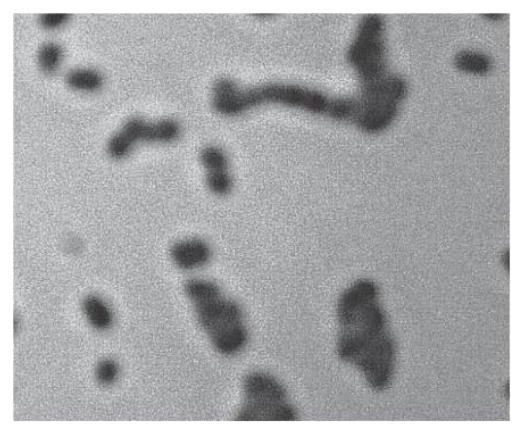

Kanamycin

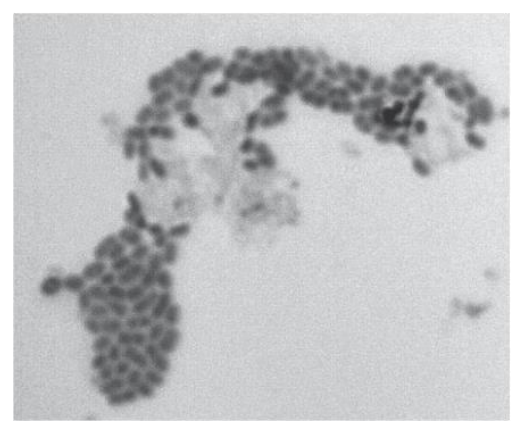

\section{Bacitracin}

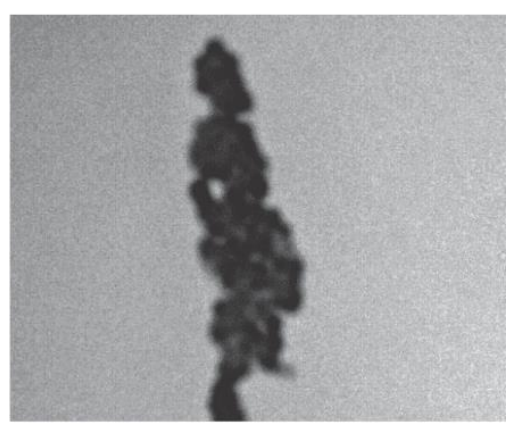

\section{Actinomycin D}

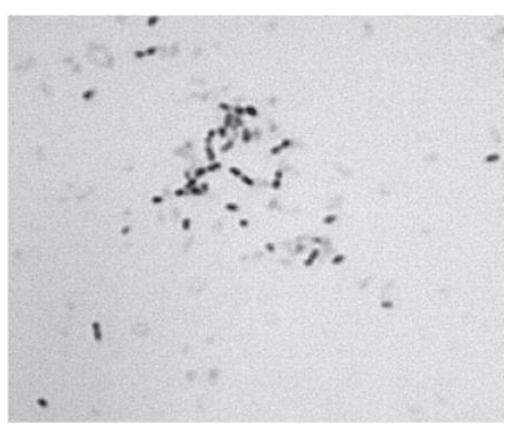

fgkc

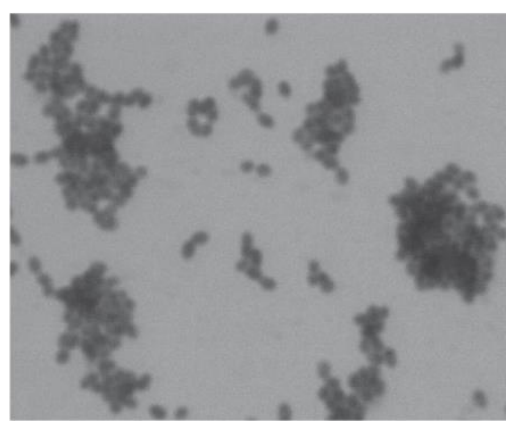

Figure 3. Phenotypic analysis of wild-type S.pneumoniae in the presence of sub-MIC (0.7x) of antibiotics with well-defined modes of action or fgkc. Cells were treated for $90 \mathrm{~min}$ with

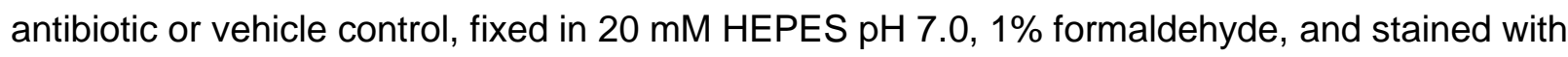
methylene blue.

Inhibition of LytB activity was investigated in a chain dispersing assay with the $\Delta / y t B$ strain and exogenously added LytB. $(14,27)$ When the $\Delta / y t B$ mutant is treated with exogenous LytB, dispersion of the chains is observed (Figure 4). When a 5-fold MIC (40 $\mu \mathrm{M})$ concentration of fgkc was added, LytB catalyzed chain dispersion was not inhibited. In vitro analysis with Remazol brilliant blue labeled PG $(14,29)$ and purified LytB confirmed these results. This lack of 
inhibition of the biochemical activity of purified LytB is inconsistent with the larger MIC observed against the $\Delta / y t B$ mutant.

wildtype

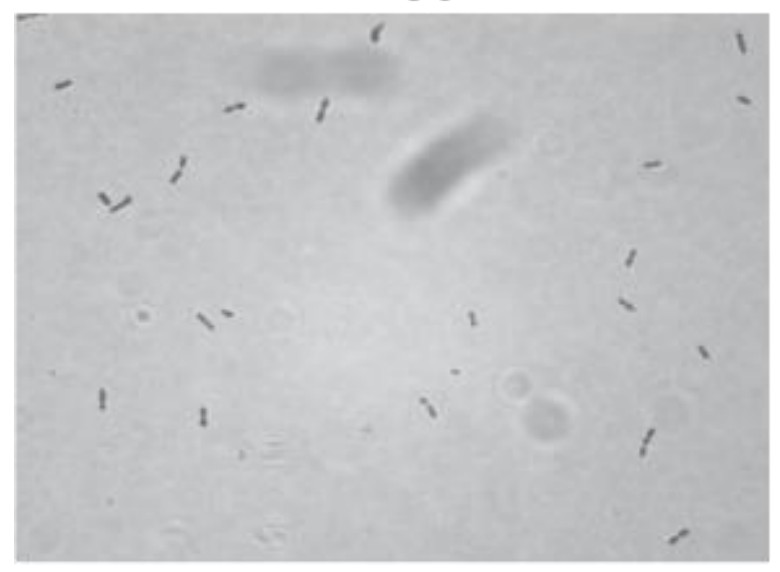

$\Delta l y t B+r L y t B$

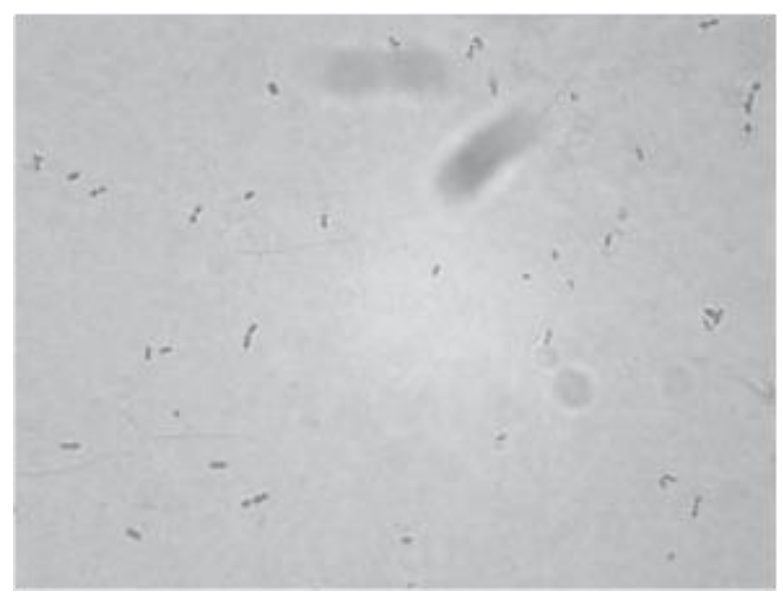

$\Delta l y t B$

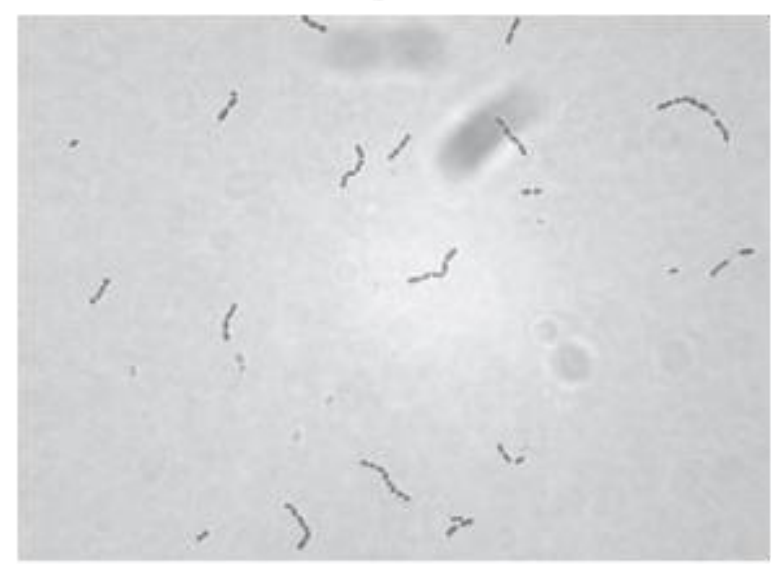

$\Delta l y t B+r L y t B+f g k c$

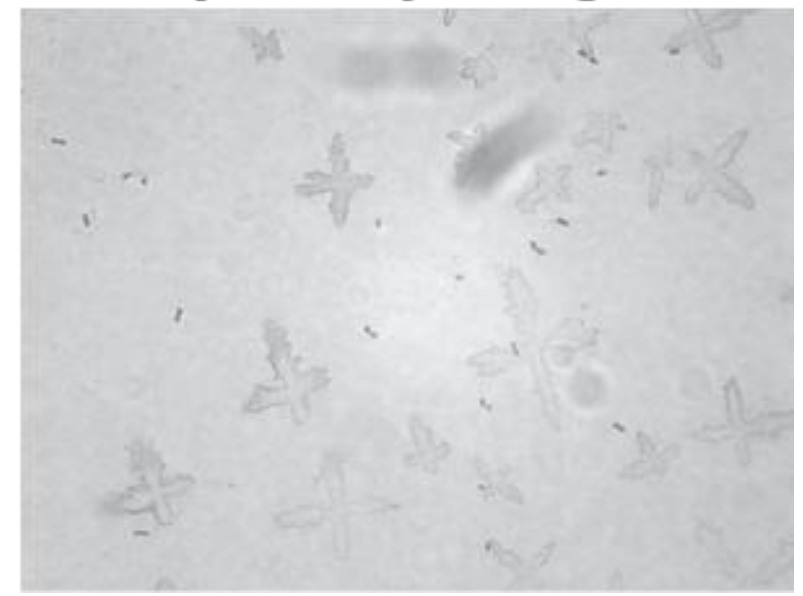

Figure 4. Chain dispersing assay with S.pneumoniae $\Delta l y t B$ strain and purified recombinant LytB (rLytB, $2 \mu \mathrm{M}$ ). In the presence of $40 \mu \mathrm{M}$ fgkc dispersion of the $\Delta l y t B$ chain phenotype is not inhibited.

This contradictory data suggests that the reduction in autolysis observed with fgkc, phenotypic, and genetic screening results are meta-phenotypes -a phenotype that result from the alteration of more than one pathways associated with the cell wall.(30) For instance, the clumping metaphenotype observed in S.pneumoniae in the presence of fgkc could be generated via a direct mechanism (e.g. inhibition of an enzyme associated with cell wall metabolism) or an indirect indirect one (e.g. alterations in autolysin expression levels, changes in metabolic flux through cell wall associated pathways). To further explore this meta-phenotype hypothesis, we looked to see if the $\triangle l y t B$ chain phenotype could be converted to the clumping phenotype observed in 
wild-type S. pneumoniae. Sub-MIC treatment of S.pneumoniae $\Delta / y t B$ with $\mathbf{f g k c}$, cefoxitin (DacA/PBP3 selective)(31, 32), or a combination of the two was examined by microscopy (Figure 5). Treatment with $0.7 x \mathrm{MIC}$ fgkc resulted in the conversion of the $\Delta / y t B$ chaining phenotype to the clumping phenotype observed when wild-type $S$. pneumoniae is treated with fgkc. This observation clearly indicates that fgkc is perturbing the cell wall phenomena observed in the $\Delta / y t B$ phenotype, suggesting a molecular target other than LytB. This observation also directly contradicts the genetic screen results in Figure $2 B$, which point to $L y t B$ as a target.

Interestingly, when fgkc was co-administered with $0.7 x$ MIC cefoxitin a disruption of the fgkcinduced clumping phenotype was observed. A further analysis of the genetic screen results showed mild decreases in sensitivity (approximately 2-fold) in the $\triangle p m p 23$ and $\triangle d a c A$ strains. Pmp23 is a putative muramidase that is required for proper localization of the Z-ring and the FtsZ-positioning protein MapZ.(33) DacA is a D,-D-carboxypeptidase (penicillin-binding protein 3) shown to be involved in cell separation. $(34,35)$

Taking this data holistically, we posit that fgkc disrupts autolytic activity associated with the cell division machinery in $S$. pneumoniae. This hypothesis is supported by reported protein interaction data that shows the three autolysins identified in the genetic screen are associated with components of the cell division complex. $(33,36,37)$ This data demonstrates that genetic knockouts of cell wall acting enzymes can be distinct from chemical knockout. This distinction has previously been observed in Mycobacterium tuberculosis shikimate biosynthesis.(38) Further, these data illustrate the complexity involved in deciphering the underlying mechanisms associated with these meta-phenotypes, and obfuscates target identification. Despite these challenges, the elucidation of the molecular target of fgkc are on-going. However, these data illustrate the promise that small molecule inhibitors to autolytic enzymes can play in furthering our understanding of peptidoglycan metabolism. 


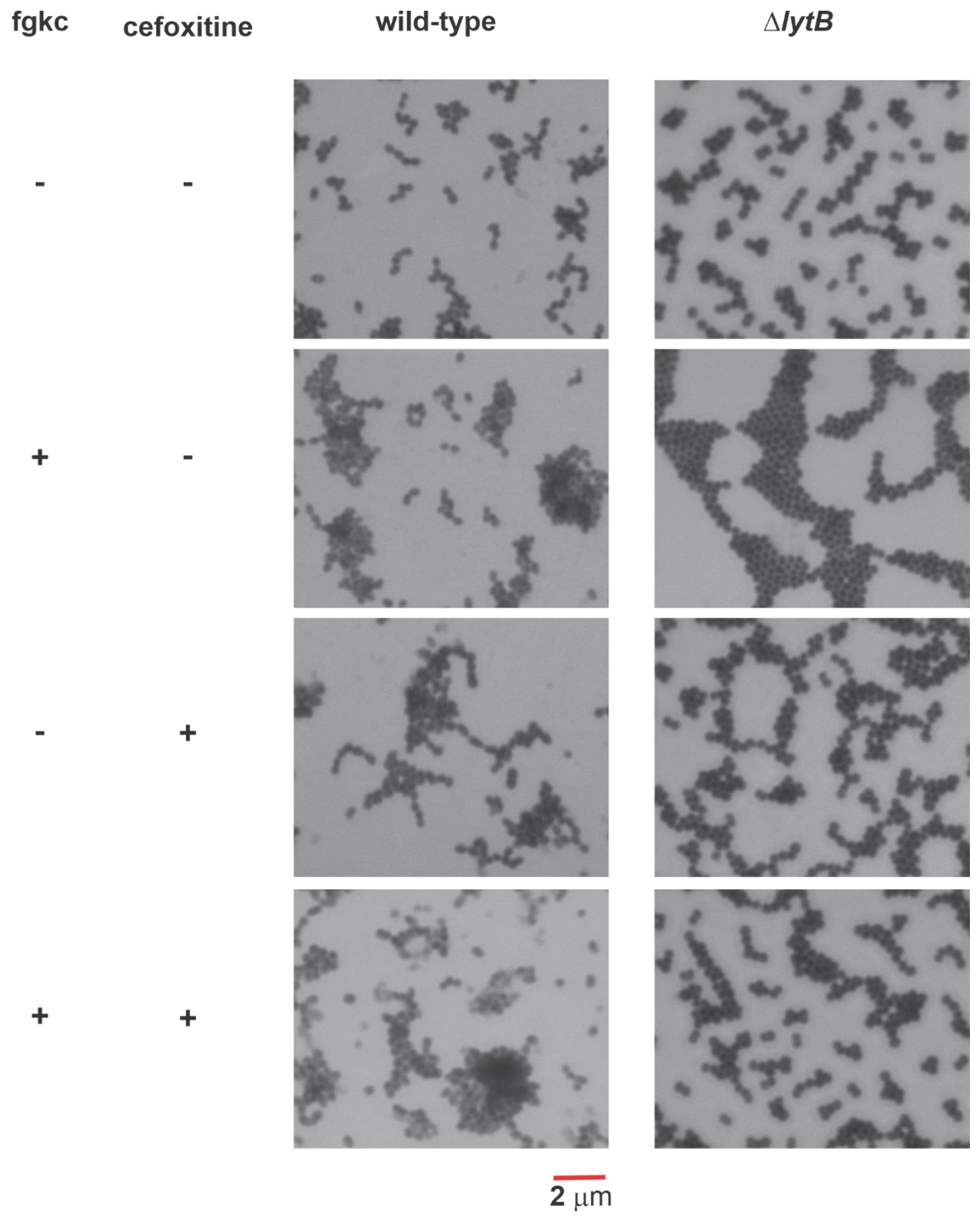

Figure 5. Morphological analysis of $S$. pneumoniae $\triangle l y t B$ mutant- (13) in the presence of subMIC fgkc, the $\beta$-lactam cefoxitin (DacA/PBP3 selective) or in combination. Cells were fixed in $1 \%$ formaldehyde, stained with methylene blue and visualized using bright field microscopy under oil immersion. 


\section{Conclusion}

The bacterial cell wall, and PG biosynthesis has provided a wealth of clinically relevant antibiotic targets. While our understanding of PG biosynthetic and cross-linking steps is fairly well established, our knowledge of the role autolytic enzymes play in the growth and maintenance of the cell wall has remained more elusive. Traditional genetic approaches to studying autolysin biological role are complicated by functional redundancy of these enzymes, where other autolysins can compensate for a loss in activity. The results presented here illustrate the complexity of PG metabolism and that phenotypes may be the manifestation of a complex interplay between associated systems. Biophysical $(3,4)$ and computational studies(5) have begun to elucidate the role of autolysins in relieving stress in the cell wall to allow for incorporation of new material into the stress bearing layer. The results with the diamide fgkc demonstrate that there are distinct differences between the genetic and chemical inactivation of cell wall acting enzymes. Collectively our data indicate that morphological, genetic, and whole cell assays (autolysis) reveal meta-phenotypes that result from the complex interaction of one or more cellular processes that are connected to cell wall metabolism. The genetic deletion of one or more autolysins disrupts the equilibrium stoichiometry of the cell wall machinery that likely results in changes to expression levels and activity to both autolytic and biosynthetic enzymes. With interest in the development of chemical biology approaches to study PG metabolism(3943) receiving renewed attention, the diamide inhibitor fgkc provides an orthogonal approach to both traditional and chemical biology approaches to studying cell wall metabolism.

\section{Acknowledgements}

Research was partially supported by the National Science Foundation (CHE2009522) and by the Rhode Island Institutional Development Award (IDeA) Network of Biomedical Research Excellence from the National Institute of General Medical Sciences of the National Institutes of Health under grant number P20GM103430 We thank J. Belval for technical assistance.

\section{References Cited}

1. Munita JM, Bayer AS, Arias CA. Evolving Resistance Among Gram-positive Pathogens. Clin Infect Dis. 2015;61(suppl 2):S48-S57. 
2. Koch AL, Doyle RJ. Inside-to-outside growth and turnover of the wall of Gram-positive rods. J Theor Biol. 1985;117:137-57.

3. Beeby M, Gumbart JC, Roux B. Architecture and assembly of the Gram-positive cell wall. Mol Microbiol. 2013;88:664-72.

4. Wheeler R, Turner RD, Bailey RG, Salamaga BI, Mesnage S, Mohamad SAS, et al. Bacterial cell enlargement requires control of cell wall stiffness mediated by peptidoglycan hydrolases. mBio. 2015;6:e00660-15.

5. Misra G, Rojas ER, Gopinathan A, et al. Mechanical consequences of cell-wall turnover in the elongation of a Gram-positive bacterium. Biophys J. 2013;104:2342-52.

6. Taguchi A, Kahne D, Walker S. Chemical tools to characterize peptidoglycan synthases. Curr Opin Chem Biol. 2019;53:44-50.

7. Welsh MA, Schaefer K, Taguchi A, Kahne D, Walker S. Direction of chain growth and substrate preferences of shape, elongation, division, and sporulation-family peptidoglycan glycosyltransferases. J Am Chem Soc. 2019;141(33):12994-7.

8. Rubino FA, Mollo A, Kumar S, Butler EK, Ruiz N, Walker S, et al. Detection of transport intermediates in the peptidoglycan flippase MurJ identifies residues essential for conformational cycling. J Am Chem Soc. 2020;142(12):5482-6.

9. Sjodt M, Brock K, Dobihal G, Rohs PDA, Green AG, Hopf TA, et al. Structure of the peptidoglycan polymerase RodA resolved by evolutionary coupling analysis. Nature. 2018;556(7699):118-21.

10. Lai GC, Cho H, Bernhardt TG. The mecillinam resistome reveals a role for peptidoglycan endopeptidases in stimulating cell wall synthesis in Escherichia coli. PLOS Genetics. 2017;13(7):e1006934.

11. Gautam A, Vyas R, Tewari R. Peptidoglycan biosynthesis machinery: a rich source of drug targets. Crit Rev Biotechnol. 2010;31:295-336.

12. Nayyab S, O'Connor M, Brewster J, Gravier J, Jamieson M, Magno E, et al. Diamide inhibitors of the Bacillus subtilis $\mathrm{N}$-acetylglucosaminidase LytG that exhibit antibacterial activity. ACS Infect Dis. 2017;3(6):421-7.

13. VanNieuwenhze MS, Brun YV, Winkler ME. Minimal peptidoglycan (PG) turnover in wild-type and PG hydrolase and cell division mutants of Streptococcus pneumoniae D39 growing planktonically and in host. J Bacteriol. 2015.

14. Bai X-H, Chen H-J, Jiang Y-L, Wen Z, Huang Y, Cheng W, et al. Structure of pneumococcal peptidoglycan hydrolase LytB reveals insights into the bacterial cell wall remodeling and pathogenesis. J Biol Chem. 2014;289:23403-16.

15. Camacho M, Portaels F, Palomino J-C. Resazurin microtiter assay plate testing of Mycobacterium tuberculosis susceptibilities to second-line drugs: rapid, simple, and inexpensive method. Antimicrob Agents Chemother. 2003.

16. Palomino J-C, Martin A, Camacho M. Resazurin microtiter assay plate: simple and inexpensive method for detection of drug resistance in Mycobacterium tuberculosis. Antimicrob Agent Chemother. 2002.

17. Czarny TL, Perri AL, French S, Brown ED. Discovery of novel cell wall-active compounds using $P$ ywaC, a sensitive reporter of cell wall stress, in the model gram-positive bacterium Bacillus subtilis. Antimicrob Agents Chemother. 2014;58:3261-9.

18. Cornett JB, Shockman GD. Cellular lysis of Streptococcus faecalis induced with triton X-100. J Bacteriol. 1978;135(1):153-60.

19. Furlan RLA, Garrido LM, Brumatti G, Amarante-Mendes GP, Martins RA, Facciotti MCR, et al. A rapid and sensitive method for the screening of DNA intercalating antibiotics. Biotechnol Lett.

2002;24:1807-13. 
20. Huang J, Shi J, Molle V, Sohlberg B, Weaver D, Bibb MJ, et al. Cross-regulation among disparate antibiotic biosynthetic pathways of Streptomyces coelicolor. Mol Microbiol. 2005;58(5):1276-87.

21. Zimmerman T, Ibrahim S. Choline Kinase, A Novel Drug Target for the Inhibition of Streptococcus pneumoniae. Antibiotics. 2017;6(4).

22. García P, González MP, García E, López R, García JL. LytB, a novel pneumococcal murein hydrolase essential for cell separation. Molecular Microbiology. 1999;31(4):1275-7.

23. Rico-Lastres P, Diez-Martinez R, Iglesias-Bexiga M, Bustamante N, Aldridge $C$, Hesek D, et al. Substrate recognition and catalysis by LytB, a pneumococcal peptidoglycan hydrolase involved in virulence. Sci Reports. 2015;5:16198.

24. Lipski A, Lipski R, Hervé M, Lombard V, Nurizzo D, Mengin-Lecreulx D, et al. Structural and biochemical characterization of the $\beta-\mathrm{N}$-acetylglucosaminidase from Thermotoga maritima: toward rationalization of mechanistic knowledge in the GH73 family. Glycobiology. 2015.

25. Horsburgh GJ, Atrih A, Williamson MP, Foster SJ. LytG of Bacillus subtilis is a novel peptidoglycan hydrolase: the major active glucosaminidase. Biochemistry. 2003;42:257-64.

26. Arrigucci R, Pozzi G. Identification of the chain-dispersing peptidoglycan hydrolase LytB of Streptococcus gordonii. PLOS ONE. 2017;12(4):e0176117.

27. De Las Rivas B, García JL, López R, García P. Purification and polar localization of pneumococcal LytB, a putative endo- $\beta-\mathrm{N}$-acetylglucosaminidase: the chain-dispersing murein hdrolase. J Bacteriol. 2002;184(18):4988-5000.

28. Klainer AS, Perkins RL. Surface manifestations of antibiotic-induced alterations in protein synthesis in bacterial cells. Antimicrob Agents Chemother. 1972;1(2):164-70.

29. Zhou R, Chen S, Recsei P. A dye release assay for determination of lysostaphin activity. Anal Biochem. 1988;171(1):141-4.

30. Martín-Galiano AJ, Yuste J, Cercenado MI, de la Campa AG. Inspecting the potential physiological and biomedical value of 44 conserved uncharacterised proteins of Streptococcus pneumoniae. BMC Genomics. 2014;15(1):652.

31. Krauss J, Hakenbeck R. A mutation in the D,D-carboxypeptidase penicillin-binding protein 3 of Streptococcus pneumoniae contributes to cefotaxime resistance of the laboratory mutant $\mathrm{C} 604$. Antimicrob Agents Chemother. 1997;41(5):936-42.

32. Kocaoglu O, Tsui H-CT, Winkler ME, Carlson EE. Profiling of $\beta$-lactam selectivity for penicillinbinding proteins in Streptococcus pneumoniae D39. Antimicrob Agents Chemother. 2015;59(6):3548-55. 33. Jacq M, Arthaud C, Manuse S, Mercy C, Bellard L, Peters K, et al. The cell wall hydrolase Pmp23 is important for assembly and stability of the division ring in Streptococcus pneumoniae. Sci Reports. 2018;8(1):7591.

34. Abdullah MR, Gutiérrez-Fernández J, Pribyl T, Gisch N, Saleh M, Rohde M, et al. Structure of the pneumococcal I,d-carboxypeptidase DacB and pathophysiological effects of disabled cell wall hydrolases DacA and DacB. Mol Microbiol. 2014;93:1183-206.

35. Schuster C, Dobrinski B, Hakenbeck R. Unusual septum formation in Streptococcus pneumoniae mutants with an alteration in the D,D-carboxypeptidase penicillin-binding protein 3. J Bacteriol. 1990;172(11):6499-505.

36. Fadda D, Santona A, D'Ulisse V, Ghelardini P, Ennas MG, Whalen MB, et al. Streptococcus pneumoniae DivIVA: localization and interactions in a MinCD-free context. J Bacteriol. 2007;189(4):128898.

37. Morlot C, Noirclerc-Savoye M, Zapun A, Dideberg O, Vernet T. The D,D-carboxypeptidase PBP3 organizes the division process of Streptococcus pneumoniae. Mol Microbiol. 2004;51(6):1641-8.

38. Ramachandran V, Singh R, Yang X, Tundugur R, Mohapatra S, Khandelwal S, et al. Genetic and chemical knockdown: a complementary strategy for evaluating an anti-infective target. Adv Appl Bioinform Chem. 2013;6:1-13. 
39. DeMeester KE, Liang H, Jensen MR, Jones ZS, D'Ambrosio EA, Scinto SL, et al. Synthesis of functionalized $\mathrm{N}$-acetyl muramic acids To probe bacterial cell wall recycling and biosynthesis. J Am Chem Soc. 2018;140(30):9458-65.

40. Liang H, DeMeester KE, Hou C-W, Parent MA, Caplan JL, Grimes CL. Metabolic labelling of the carbohydrate core in bacterial peptidoglycan and its applications. Nature Commun. 2017;8:15015.

41. Wang Y, Lazor KM, DeMeester KE, Liang H, Heiss TK, Grimes CL. Postsynthetic modification of bacterial peptidoglycan using bioorthogonal $\mathrm{N}$-acetylcysteamine analogs and peptidoglycan $\mathrm{O}$ acetyltransferase B. J Am Chem Soc. 2017;139(39):13596-9.

42. Lebar MD, May JM, Meeske AJ, Leiman SA, Lupoli TJ, Tsukamoto H, et al. Reconstitution of peptidoglycan cross-linking leads to improved fluorescent probes of cell wall synthesis. J Am Chem Soc. 2014;136(31):10874-7.

43. Dik DA, Zhang N, Chen JS, Webb B, Schultz PG. Semisynthesis of a bacterium with non-canonical cell-wall cross-links. J Am Chem Soc. 2020. 